\title{
Pharmacological reversal of advanced glycation end-product-mediated protein crosslinking
}

\author{
P. U Irich, X . Z hang \\ The Picower Institute for Medical Research, Manhasset, New York, USA
}

It is well known that glucose reacts non-enzymatically with protein amino groups with eventual formation of covalent protein-protein crosslinks, which are called advanced glycation end-products (AGEs) [1]. Much work has been done to establish a causative association between this process and many of the pathological sequelae observed in diabetes mellitus, including LDL modification and the vascular changes which lead to atherosclerosis [2,3]. AGE crosslinking also occurs between tissue proteins and AGE-containing peptide fragments formed from protein turnover. Such reactive AGE-peptides, now called glycotoxins, are normally cleared by the kidneys, but they build up in the serum in conditions such as diabetic kidney failure, and their random covalent attachment to proteins may cause widespread tissue damage.

In the early 1980s we suspected that reactive carbonyls were involved in the mechanism of crosslinking and tissue damage by AGEs, and so we developed the carbonyl-blocking agent, aminoguanidine, as a new pharmaceutical for inhibition of the development of diabetic complications [4]. Aminoguanidine is now in Phase 2/Phase 3 clinical trials under the generic name pimagedine. However, development of improved agents for preventing or reversing this process in vivo has been hampered by a lack of detailed knowledge of the chemical nature of the principal crosslinking structures. The crosslinking structures identified to date are thought on the basis of chemical considerations and spectroscopic data to constitute only a small fraction of the AGE crosslinking which occurs in vivo, with the major crosslinking structures still unidentified [5].

Corresponding author: Dr. P. Ulrich, The Picower Institute for Medical Research, 350 Community Drive, Manhasset, NY 11030, USA

A bbreviations: AGE, Advanced glycation end products; PTB, phenacyl thiazolium bromide.
We have now found that certain substituted thiazolium salts can actually break covalent AGE crosslinks and return the affected proteins to the uncrosslinked state both in vitro and in vivo [6]. In addition to demonstrating the unexpected new possibility of pharmacological reversal of clinically relevant AGE crosslinking, this cleavage reaction provides new chemical information about the nature of the major AGE crosslinks by virtue of the unusual chemistry of which thiazolium salts are capable. In collaboration with workers at Alteon Inc., (Ramsey, N.J., USA) a number of thiazolium derivatives have been prepared and studied both for the inhibition and the reversal of AGE-crosslinking. One compound of particularly high activity, phenacyl thiazolium bromide (PTB), has been studied in detail. PTB rapidly cleaves a model diketone, phenylpropanedione, with release of benzoic acid.

In an in vitro assay, AGE-BSA was allowed to crosslink to collagen which had been coated onto microtitre wells, and unreacted AGE-BSA was washed away. PTB released BSA that had been crosslinked to the collagen in a dose- and time-dependent manner. Aminoguanidine is not able to release crosslinked BSA in this assay.

Normal rat tail tendon collagen yields electrophoresible fragments on digestion with cyanogen bromide. However, tail collagen from diabetic rats releases almost no such fragments on $\mathrm{CNBr}$ treatment. This is because of extensive additional crosslinking due to AGE formation. This effect is almost completely reversed on pre-treatment of the isolated collagen with PTB prior to cyanogen bromide digestion. Such a reversal is not seen on pre-treatment with aminoguanidine.

In diabetic rats IgG becomes covalently bound to erythrocyte membrane proteins through AGE reactions. However, in diabetic rats which received PTB at $10 \mathrm{mg} / \mathrm{kg}$ four times per day for up to 4 weeks, 
release of erythrocyte-bound $\mathrm{IgG}$ occurred, reaching a level of $60-70 \%$ cleavage. In vitro, treatment of diabetic rat erythrocytes with PTB causes IgG binding to revert to control levels.

There is evidence that AGE crosslinking may contribute to amyloid nucleation and increased amyloid deposition in Alzheimer's disease. In vitro, glucose treatment of $\beta$-amyloid peptide causes AGE formation and enhances aggregation. Treatment of aggregated fibrillar AGE $\beta$-amyloid with PTB results in significant disaggregation of the amyloid, as determined by gel electrophoresis of radiolabelled material, and electron microscopy.

Our proposed mechanism for AGE crosslink cleavage by thiazolium salts involves dicarbonyl containing crosslinks derived from the early glycation adduct, the Amadori product, a fructosamine derivative which forms by rearrangement of the initially formed Schiff-base adduct of glucose with protein amino groups. The Amadori product can undergo a number of subsequent rearrangements which lead to the formation of $\alpha$-dicarbonyl compounds. We have become interested in the little studied amino dicarbonyl adducts, the Amadori dione [7, 8] and the Amadori ene-dione [9] (Fig.1). These potentially important AGE intermediates have been neglected due to their instability and difficulty of synthesis. They were very interesting to us because they contain diketone and ene-dione reactive centres which could in theory undergo crosslinking with suitably reactive side chains of other proteins. It was to destroy crosslink precursors such as these that we chose to study thiazolium salts, which as a class are known to be able to chemically cleave $\alpha$-diketones by breaking the bond between the carbonyl groups [10].

In particular we were interested in the Amadori ene-dione because it contains an enone structure of a type which is known to react covalently with various nucleophilic peptide side chains such as lysine, cysteine, and histidine, which would lead to formation of $\alpha$-dicarbonyl AGE crosslinks of the type which would be cleavable by thiazolium salts.

Figure 1 shows the way in which the thiazolium salt PTB could cleave such a crosslink. PTB was designed to have two nucleophilic centres, one on the ring and one on the side chain. Reaction of these with the electrophilic $\alpha$-dicarbonyl would give a cyclic intermediate which could rearrange with cleavage of the carbon-carbon bond between the carbonyls, by analogy with the enzymatic reactions of thiamine. The crosslink fragment attached to the side chain would then deacylate to give a carboxylic acid, resulting in breakage of the crosslink. Cleavage of the other crosslink fragment from PTB appears to be a slower step at physiological $\mathrm{pH}$, but we are currently working on designing better agents to increase the rate of this step, which would allow the cleavage process to be catalytic with regeneration of the cleavage agent.

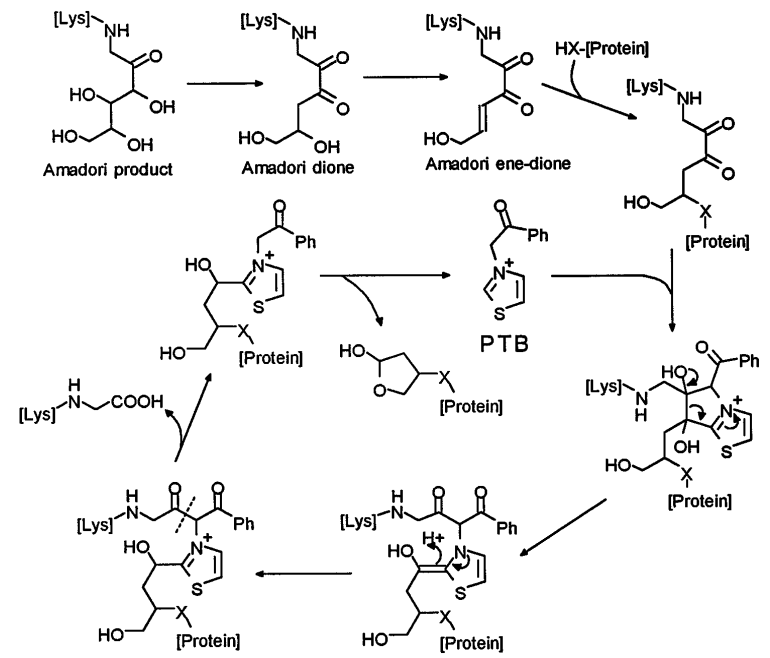

Fig. 1. Proposed mechanism of formation of $\alpha$-dicarbonyl AGE crosslinks and their cleavage by the thiazolium salt PTB

To verify the viability of this mechanism under physiological conditions, it is desirable to have a way of chemically preparing such ene-dione derived AGE crosslinks for study in vitro. The formation of Amadori ene-dione by two successive dehydrations of the Amadori product (Fig. 1) is not efficient or rapid enough to provide a convenient source of pure ene-dione for in vitro studies.

We therefore developed a synthetic route to prepare a novel Amadori ene-dione precursor with improved leaving groups, toluenesulfonate esters, at the dehydration sites. Incubation of this disulfonate precursor at $\mathrm{pH} 7.4$ and $37^{\circ}$ in the absence of other nucleophiles leads to cyclization products indicating the intermediacy of Amadori ene-dione [11]. We thus now have a tool which will allow us to study model ene-dione crosslinking reactions for elucidation of the mechanism of AGE crosslink breakage by PTB and related agents.

In summary, we have discovered that agents designed to cleave $\alpha$-dicarbonyl compounds can break protein-protein advanced glycation crosslinks. Such agents may lead to the development of new drugs for clinical reversal of diabetes complications. We have proposed a novel mechanism by which $\alpha$-dicarbonyl containing AGE crosslinks might form, and have developed chemical tools for studying this theory.

\section{References}

1. Ledl F, Schleicher E (1990) New aspects of the Maillard reaction in foods and in the human body. Angew Chem Int Ed Engl 29: 565-594

2. Horiuchi S (1996) Advanced glycation end products (AGE)-modified proteins and their potential relevance to atherosclerosis. Trends Cardiov Med 6: 163-168

3. Palinski W, Koschinsky T, Butler SW, Miller E, Vlassara H, Cerami A, Witztum JL (1995) Immunological evidence for 
the presence of advanced glycosylation end products in atherosclerotic lesions of euglycemic rabbits. Arteriosc Thromb Vasc Biol 15: 571-582

4. Brownlee M, Vlassara H, Kooney A, Ulrich P, Cerami A (1986) Aminoguanidine prevents diabetes-induced arterial wall protein cross-linking. Science 232: 1629-1632

5. Dyer DG, Blackledge JA, Katz BM et al. (1991) The Maillard reaction in vivo. Zeitschr Ernahrungswiss 30: 29-45

6. Vasan S, Zhang X, Zhang X et al. (1996) An agent cleaving glucose-derived protein crosslinks in vitro and in vivo. Nature 382: $275-278$

7. Huber B, Ledl F (1990) Formation of 1-amino-1,4-dideoxy2,3-hexodiuloses and 2-aminoacetylfurans in the Maillard reaction. Carbohyd Res 204: 215-220
8. Chen HJC, Cerami A (1993) Mechanism of inhibition of advanced glycosylation by aminoguanidine in vitro. J Carbohyd Chem 12: 731-742

9. Estendorfer S, Ledl F, Severin T (1990) Formation of an aminoreductone from glucose. Angew Chem Int Ed Engl 29: $536-537$

10. Vovk AI, Murav'eva IV, Yasnikov AA (1985) Mechanism of the cleavage of acetylbenzoyl in methanol catalyzed by thiazolium salts. Ukr Khim Zh, Russ Ed 51: 521-525

11. Zhang X, Ulrich P (1996) Directed approaches to reactive Maillard intermediates: Formation of a novel 3-alkylamino-2-hydroxy-4-hydroxymethyl-2-cyclopenten-1-one ("cypentodine"). Tetrahedron Lett 37: 4667-4670 